\title{
Application of low-energy theorems to NN scattering at unphysical pion masses
}

\author{
V. Baru* \\ Institut für Theoretische Physik II, Ruhr-Universität Bochum, D-44780 Bochum, Germany and \\ Institute for Theoretical and Experimental Physics, B. Cheremushkinskaya 25, 117218 Moscow, \\ Russia \\ E-mail: vadimbatp2.rub.de \\ E. Epelbaum
}

Institut für Theoretische Physik II, Ruhr-Universität Bochum D-44780 Bochum, Germany E-mail: evgeny. epelbaumeruhr-uni-bochum. de

\author{
A. A. Filin \\ Institut für Theoretische Physik II, Ruhr-Universität Bochum D-44780 Bochum, Germany \\ E-mail: arseniy.filinetp2.ruhr-uni-bochum.de

\section{J. Gegelia} \\ Institute for Advanced Simulation, Institut für Kernphysik and Jülich Center for Hadron Physics, \\ Forschungszentrum Jülich, D-52425 Jülich, Germany and \\ Tbilisi State University, 0186 Tbilisi, Georgia \\ E-mail: jgegelia@hotmail.com
}

\begin{abstract}
The low-energy theorems (LET) for NN scattering provide important relations between the coefficients in the effective-range expansion (ERE) of the amplitude, which are governed by the long-range part of the potential. In this work we extend the LET to the case of unphysical pion masses. It is emphasized that correlations provided by LET can be used as nontrivial consistency checks for lattice calculations. As an example, we use the $M_{\pi}$ dependence of the effective range suggested in the recent lattice study by NPLQCD collaboration [1] as input to predict the $M_{\pi}$ dependence of the binding energy and the shape parameters.
\end{abstract}

The 8th International Workshop on Chiral Dynamics, CD2015 ***

29 June 2015 - 03 July 2015

Pisa,Italy

\footnotetext{
* Speaker.
} 


\section{Introduction}

The understanding of certain fine-tuning in the parameters of the Standard Model is of particular importance in modern hadron and nuclear physics. The fine-tuning refers in particular to the very specific values of the light-quark masses so that only very small variations in these parameters would still allow our Universe to exist in the form it has now, see Ref. [2] for a recent review. In particular, the proximity of the excited state of ${ }^{12} \mathrm{C}$ - the Hoyle state - to the triple alpha-particle threshold is known to be crucial for the enhanced resonance formation of the life-important elements ${ }^{12} \mathrm{C}$ and ${ }^{16} \mathrm{O}$ in stars. The dependence of the excitation energy of the Hoyle state on the light-quark masses was analysed recently within ab initio nuclear lattice simulations [3, 4]. It was understood that the variation of the excitation energy by $\pm 100 \mathrm{KeV}$ which is still consistent with the sufficient abundances of ${ }^{12} \mathrm{C}$ and ${ }^{16} \mathrm{O}$ [5] can be maintained only if the quark masses are varied by a few per cents. By far the dominant source of the theoretical uncertainty in this calculation is related to the lack of knowledge of the quark mass dependence of the nuclear force or, more precisely, of the nucleon-nucleon (NN) S-wave scattering lengths. The quark mass dependence of the nuclear force plays an important role for investigating the time dependence of the fundamental constants beyond the Standard Model. In particular, the theory of Big Bang nucleosynthesis (BBN) and the observed primordial abundances allow one to estimate a possible quark mass variation at the time of BBN [6,7], provided that the quark mass dependence of the S-wave NN scattering lengths and the binding energies of the light nuclei from ${ }^{3} \mathrm{He}$ to ${ }^{7} \mathrm{Be}$ is known.

In recent years, we have witnessed a significant progress in the lattice QCD calculations which are considered as the primary source of information about the light-quark or equivalently the pion mass dependence of the nuclear observables. In particular, the fully dynamical calculations at relatively low unphysical pion masses $M_{\pi} \simeq 300 \mathrm{MeV}$ (or a bit higher) have now become possible (see e.g. Refs. $[8,9,10]$ ). The connection of these results with the physical world can be provided by chiral effective field theory (chiral EFT) which at these pion masses might still be within its range of validity. Notice that not only the binding energies of nuclear observables but also the NN scattering data - phase shifts and effective range parameters - are calculable on lattice, see Refs. $[10,1]$ for the recent studies of the ${ }^{1} S_{0}$ and ${ }^{3} S_{1}$ channels by NPLQCD collaboration at $M_{\pi} \simeq 450$ and $800 \mathrm{MeV}$ and Ref.[11] for the analysis of the higher partial waves at $M_{\pi} \simeq 800 \mathrm{MeV}$ by CalLat collaboration. Interestingly, the general trend of different lattice calculations $[8,9,10,1,12]$ exhibits a stronger attraction both in the ${ }^{1} S_{0}$ and ${ }^{3} S_{1}$ channels when one goes away from the physical point. Consequently, both the deuteron and the dineutron systems in these works are found to be bound at unphysical pion masses with the deuteron binding energy larger than the physical one. These results are, however, not supported by the HAL QCD collaboration which finds no bound states in these channels for pion masses ranging from 469 to $1171 \mathrm{MeV}$ [13]. It should be noted that unlike the other studies, a different approach by making use of the $\mathrm{NN}$ potential at the intermediate step of the calculation is employed by the HAL QCD collaboration. The puzzle is getting even more intriguing given that the chiral EFT calculations generally suggest less attraction at $M_{\pi}$ larger than the physical value $[14,15,16,7,17]$. These calculations, however, rely on naturalness or resonance saturation estimates for the $M_{\pi}$-dependent $4 N$ contact operator at next-to-leading order.

In this contribution we follow the lines of Ref.[18] to argue that the low-energy theorems in NN scattering can be useful for providing consistency checks of both lattice results and theory approaches. We first discuss the LET in the physical world and then generalise them to unphysical $M_{\pi}$. 


\section{Low-energy theorems}

Long-range interactions are responsible for the near-threshold left-hand singularities of the partial wave scattering amplitude and control its energy dependence. In particular, they impose correlations between the coefficients in the effective range expansion which can be regarded as low-energy theorems [19, 20, 21, 22, 23].

Consider two non-relativistic particles of mass $m$ interacting via some non-singular potential of the finite range. The corresponding $S$-matrix for an uncoupled channel with the zero orbital angular momentum can be written in terms of the $T$-matrix as ${ }^{1}$

$$
S=e^{2 i \delta(k)}=1-i\left(\frac{k m}{8 \pi^{2}}\right) T(k),
$$

where $k$ denotes the scattering momentum in the center-of-mass system $(\mathrm{cms})$. The scattering amplitude $T$ in turn can be expressed in terms of the so-called effective range function $F(k) \equiv$ $k \cot \delta(k)$ via

$$
T(k)=-\frac{16 \pi^{2}}{m} \frac{1}{F(k)-i k} .
$$

Contrary to the scattering amplitude, the effective range function does not possess the kinematic unitarity cut and is a real meromorphic (i.e. analytic except for poles) function of $k^{2}$ near the origin $k=0[25,26]$. It can, therefore, be Taylor-expanded about the origin leading to the well-known effective range expansion which has the form ${ }^{2}$

$$
k \cot \delta(k)=-\frac{1}{a}+\frac{1}{2} r k^{2}+v_{2} k^{4}+v_{3} k^{6}+v_{4} k^{8}+\ldots,
$$

where $a$ and $r$ refer to the scattering length and the effective range, respectively, while $v_{i}$ denote the so-called shape parameters. The convergence radius of the ERE is restricted by the lowest-lying left-hand singularity associated with the potential. In particular, the longest-range part of the NN force is due to the one-pion-exchange potential (OPEP), which has the left-hand cut starting from the branch point at the cms momenta $k= \pm i M_{\pi} / 2$. The ERE for NN scattering is therefore expected to converge up to about $70 \mathrm{MeV}$ cms momenta or laboratory energies $\left|E_{\text {lab }}\right| \sim M_{\pi}^{2} /\left(2 m_{N}\right)=10.5$ $\mathrm{MeV}$.

The framework of the ERE can be generalized to the case in which the potential is given by a sum of long-range $\left(r_{L} \sim M_{L}^{-1}\right)$ and short-range $\left(r_{S} \sim M_{S}^{-1} \ll M_{L}^{-1}\right)$ potentials, $V_{L}$ and $V_{S}$, respectively [28]. The main idea of Ref. [28] is to keep the long-range physics explicitly, in particular the left-hand cuts associated with the long-range potential $V_{L}$. Then, one can introduce the modified effective range function $F^{M}$ determined by the short-range interaction $V_{S}$ which can be expressed in terms of the phase shift corresponding to the full potential $V_{L}+V_{S}$ and the quantities associated with the long-range interaction (labelled by $L$ )

$$
F^{M}\left(k^{2}\right) \equiv R^{L}(k)+\frac{k}{\left|f^{L}(k)\right|} \cot \left[\delta(k)-\delta^{L}(k)\right] .
$$

\footnotetext{
${ }^{1}$ Generalizations to the nonzero orbital angular momenta and to the coupled channel case are straightforward, see e.g. Refs. [24, 18].

${ }^{2} \mathrm{We}$ assume here that the phase does not cross zero in the region of validity of the ERE. If this is the case the Taylor expansion should be replaced, e.g. by the Pade approximants [27].
} 
where $\left.f^{L}(k) \equiv f^{L}(k, r)\right|_{r=0}$ denotes the Jost function corresponding to the Jost solution of the Schrödinger equation $f^{L}(k, r)$ for the potential $V_{L}$ while $R^{L}(k)$ is its derivative $R^{L}(k)=\lim _{r \rightarrow 0}\left[\frac{d}{d r} \frac{f^{L}(k, r)}{f^{L}(k)}\right]$. Further, $\delta^{L}(k)$ denotes the phase shift for the potential $V_{L}$. Unlike the ordinary ERE, $F^{M}\left(k^{2}\right)$ is a real meromorphic function in a much larger region set by $r_{S}^{-1}$. In particular, for Yukawa-type potentials, the region in which the modified effective range function is meromorphic is set by $|k|<M_{S} / 2$. Similarly to the ERE, one can Taylor expand the function $F^{M}\left(k^{2}\right)$ near the origin via

$$
F^{M}\left(k^{2}\right)=-\frac{1}{a^{M}}+\frac{1}{2} r^{M} k^{2}+v_{2}^{M} k^{4}+v_{3}^{M} k^{6}+v_{4}^{M} k^{8}+\ldots
$$

This expansion is referred to as the modified effective range expansion (MERE).

We are now in the position to clarify the meaning of the LET. Inverting Eq. (2.4) one can calculate the physical phase shift $\delta$ in terms of the known "long-range" quantities and the short-range physics systematically parameterized through the MERE

$$
k \cot \delta=\frac{\left|f^{L}(k)\right|\left(F^{M}\left(k^{2}\right)-R^{L}(k)\right) k \cot \delta^{L}(k)-k^{2}}{\left|f^{L}(k)\right|\left(F^{M}\left(k^{2}\right)-R^{L}(k)\right)+k \cot \delta^{L}(k)}
$$

For example, at leading order (LO), the modified effective range function is approximated as $F_{l}^{M}\left(k^{2}\right) \simeq-1 / a^{M}$. Thus, using a single piece of information about the short-range interaction in form of $a^{M}$ or, equivalently, the usual scattering length $a$ allows one to predict all coefficients in the ERE

$$
r=\frac{\alpha\left(a M_{L}\right)}{M_{L}}, v_{2}=\frac{\beta\left(a M_{L}\right)}{M_{L}^{3}}, v_{3}=\frac{\gamma\left(a M_{L}\right)}{M_{L}^{5}}, v_{4}=\frac{\delta\left(a M_{L}\right)}{M_{L}^{7}}, \ldots
$$

where $\alpha, \beta, \gamma, \delta, \ldots$ are known coefficients. These predictions are accurate up to corrections emerging from the second term in the MERE, i.e. $r^{M}$. Using $a^{M}$ and $r^{M}$ as input at NLO, or equivalently the physical scattering length and the effective range, one can improve predictions for the shape parameters including the corrections $\sim r_{M} / r_{L} \simeq M_{L} / M_{S}$. The resulting correlations between the subthreshold parameters at each fixed order in this expansion are regarded as LET. For an example of an exactly solvable model, see Ref. [21].

\section{Application of low-energy theorems to NN scattering}

The longest range part of the NN force is due to the OPE potential so that the LET in NN scattering are expected to be governed by the left hand cut generated by the OPEP. Further, the OPEP is singular at small distances and requires regularization and renormalization. Therefore instead of using the quantum mechanical approach discussed in the previous section, for the NN system we formulate the LET within the modified framework of Weinberg chiral EFT [29, 17]. Since the correlations between the ERE parameters are inherently long-range phenomena, after renormalization the results should be model and regularization scheme independent.

In particular, to find the scattering amplitude $T$ we solve the Lippmann-Schwinger type integral equation introduced by Kadyshevsky [30] which, for the case of the fully off-shell kinematics, takes the form

$$
T\left(p_{0}, \vec{p}^{\prime}, \vec{p}\right)=V\left(\vec{p}^{\prime}, \vec{p}\right)+\int d^{3} q V\left(\vec{p}^{\prime}, \vec{q}\right) G\left(p_{0}, q\right) T\left(p_{0}, \vec{q}, \vec{p}\right),
$$


where $G\left(p_{0}, q\right)$ is the free Green function

$$
G\left(p_{0}, q\right)=\frac{m_{N}^{2}}{2(2 \pi)^{3}} \frac{1}{\left(\vec{q}^{2}+m_{N}^{2}\right)\left(p_{0}-\sqrt{\vec{q}^{2}+m_{N}^{2}}+i \varepsilon\right)}
$$

Further, $\vec{p}\left(\vec{p}^{\prime}\right)$ is the incoming (outgoing) three-momentum of the nucleon in cms, $p_{0}=\sqrt{\vec{k}^{2}+m_{N}^{2}}$ with $m_{N}$ denoting the nucleon mass and $\vec{k}$ being the corresponding three-momentum of an incoming (on-mass-shell) nucleon. The potential at LO consists of the OPEP and one contact term in each of the two S-wave channels $\left({ }^{1} S_{0}\right.$ and $\left.{ }^{3} S_{1}\right)$

$$
V_{\mathrm{LO}}=-\frac{g_{A}^{2}}{4 F_{\pi}^{2}} \frac{\vec{\sigma}_{1} \cdot \vec{q} \vec{\sigma}_{2} \cdot \vec{q}}{\vec{q}^{2}+M_{\pi}^{2}} \tau_{1} \cdot \tau_{2}+C_{0}, \quad C_{0}=C_{S}+C_{T} \vec{\sigma}_{1} \cdot \vec{\sigma}_{2}
$$

where $\vec{q} \equiv \vec{p}^{\prime}-\vec{p}$ is the momentum transfer, $\vec{\sigma}_{i}\left(\tau_{i}\right)$ denote the spin (isospin) Pauli matrices of the nucleon $i$, while $g_{A}$ and $F_{\pi}$ refer to the axial vector coupling of the nucleon and pion decay constant, respectively. As discussed in Ref. [29], the LO potential in the applied framework is exactly renormalizable ${ }^{3}$, that is all divergencies appearing due to iterations of the OPEP can be absorbed into the redefinition of the contact term $C_{0}$ at LO. As a consequence, the cutoff in the integral equation can be put to infinity, so that no finite cutoff artifacts can affect the LET.

In Fig. 1 we demonstrate the results for the LET in the spin triplet channel where the LET are expected to work best due to the strong tensor force in the OPEP, see also Refs. [18, 32] for the discussion of the spin singlet case. In particular, we adjust the contact term to reproduce the scattering length $a$ which is the only input quantity at LO. Then, the LET allow one to predict the values of the effective range and the shape parameters for each given value of $a$, as discussed in the previous section. As can be seen from Fig 1, for the physical value of $a$ the LET exhibit a very good agreement with the empirical values extracted from the Nijmegen PWA [24], see also Table 1. The accuracy in the extracted parameters (cf. e.g. the effective range in the first and third rows of Table 1) appears to be even better than one could naively expect from the ratio of energies corresponding to the first left-hand cut included due to OPE and the second one not considered explicitly at this order. The reason for the better-than-naively-expected convergence of the LET can be accounted for by the fact that iterations of OPE generate also the large portion of the lefthand cut contributions from two- and multiple-pion exchanges.

At NLO a subleading short-range interaction with two space derivatives starts to contribute whose strength can be adjusted to reproduce the experimental value of the effective range. In this way this subleading short-range term accounts for the presence of additional degrees of freedom (e.g. irreducible two-pion exchanges) which are integrated out in our approach but may affect the discontinuity along the more distant left-hand cuts. Given the arguments formulated above, the results for the LET are insensitive to details of the short-range interaction once its strength is adjusted to reproduce the physical observable. It appears to be convenient to employ resonance saturation to model higher-order contact interactions by means of a heavy-meson-exchange.

$$
V_{\mathrm{NLO}}=V_{1 \pi}(\vec{q})+C_{0}+\beta \frac{\vec{\sigma}_{1} \cdot \vec{q} \vec{\sigma}_{2} \cdot \vec{q}}{\vec{q}^{2}+M^{2}}
$$

\footnotetext{
${ }^{3}$ For the recent extension of the approach to $\bar{D} D^{*}$ scattering see Ref. [31] where chiral extrapolations of the $X(3872)$ binding energy were discussed.
} 


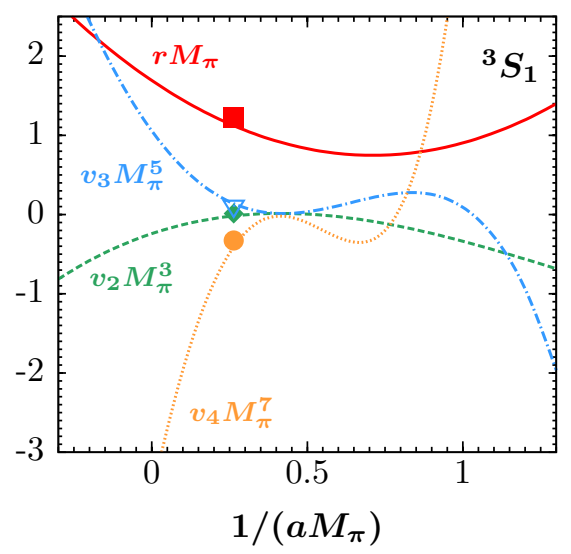

Figure 1: Correlations between the inverse scattering length $a^{-1}$, effective range $r$ and the first three shape parameters $v_{2}, v_{3}$ and $v_{4}$ induced by the one-pion exchange interaction in the ${ }^{3} \mathrm{~S}_{1}$ channel. Solid rectangles, diamonds, open triangles and circles correspond to the values of $r, v_{2}, v_{3}$ and $v_{4}$, respectively, extracted from the Nijmegen partial wave analysis $[33,34]$. The results correspond to the Blatt-Biedenharn parameterization of the S-matrix [35].

\begin{tabular}{lrrrrr}
\hline Neutron-proton ${ }^{3} \mathbf{S}_{1}$ partial wave & $a[\mathrm{fm}]$ & $r[\mathrm{fm}]$ & $v_{2}\left[\mathrm{fm}^{3}\right]$ & $v_{3}\left[\mathrm{fm}^{5}\right]$ & $v_{4}\left[\mathrm{fm}^{7}\right]$ \\
\hline LO [29] & fit & 1.60 & -0.05 & 0.82 & -5.0 \\
NLO [18] & fit & fit & 0.06 & 0.70 & -4.0 \\
\hline \hline Empirical values [24] & 5.42 & 1.75 & 0.04 & 0.67 & -4.0 \\
\hline \hline NLO KSW [19] & fit & fit & -0.95 & 4.6 & -25
\end{tabular}

Table 1: Effective-range parameters in the ${ }^{3} S_{1}$ partial wave in the Blatt-Biedenharn parameterization of the S-matrix [35]. The first two rows show the effective range parameters extracted from the LET at LO and NLO, respectively. The third row corresponds to the results of the Nijmegen PWA [24]. The last row shows the predictions obtained within the KSW framework [36] which relies upon a perturbative treatment of the OPEP. These results are taken from Ref. [19] and show very large deviations from the empirical values.

where the heavy-meson mass $M$ is set $M=700 \mathrm{MeV}$ and the strength $\beta$ is adjusted to reproduce the empirical value of the effective range. Indeed, the deviation in the results caused by using the sigma-like scalar potential instead of the tensor one in Eq. (3.4) for the sub-leading short-range interaction is negligibly small. The predicted values of the shape parameters after fixing the values of $C_{0}$ and $\beta$ are listed in Table 1 and, as expected, show a clear improvement as compared with the LO results. In fact, the NLO LET appear to be accurate at the level of a few percent (except for $v_{2}$ which is unnaturally small).

\section{LET for NN scattering: generalization to unphysical pion masses}

Generalization of LET to unphysical pion masses is straightforward:

- The main dynamical effect of changing the pion mass in the OPEP corresponds to shifts of the branch points of all left-hand cuts.

- The discontinuity across the left-hand cuts changes when the ratio $g_{A} / F_{\pi}$ in the OPEP is varied with $M_{\pi}$.

- The discontinuity across the left-hand cuts also feels the variation with $M_{\pi}$ of the nucleon mass in the integral equation (3.1). 

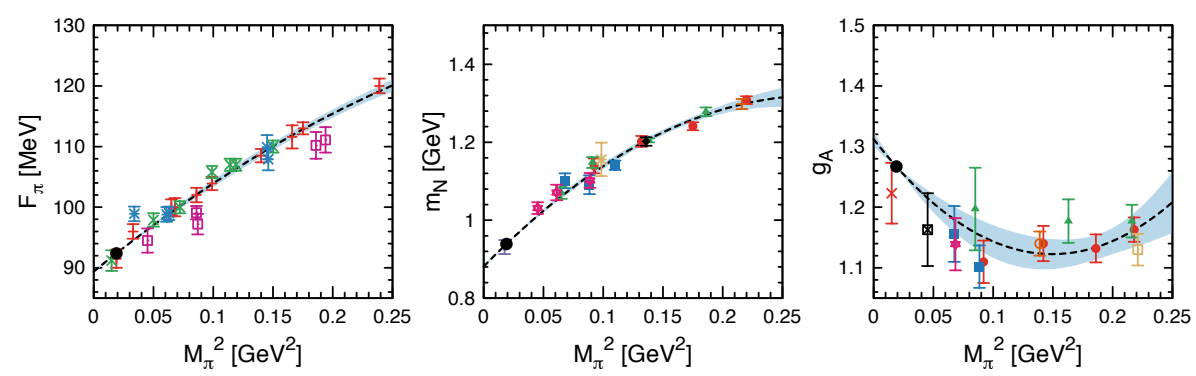

Figure 2: Quadratic polynomial regression fits to lattice QCD data for the pion decay constant $F_{\pi}$, nucleon mass $m_{N}$ and the nucleon axial-vector coupling constant $g_{A}$. Lattice data for $F_{\pi}, m_{N}$ and $g_{A}$ are from Refs.[37, 38]. Filled circles without error bars show the experimentally measured values. The dashed lines depict our fit results to the lattice data and the experimental values while the shaded bands correspond to the $67 \%$ confidence levels of the interpolations.

To account for the last two effects in a way that minimizes the theoretical uncertainty, we make use of the lattice-QCD results for the $M_{\pi}$ dependence of $g_{A}, F_{\pi}$ and $m_{N}$, see Fig. 2. In particular, we performed quadratic polynomial regression fits (as functions of $M_{\pi}^{2}$ ) of lattice-QCD data for pion masses up to $M_{\pi}=500 \mathrm{MeV}$, as shown in Fig. 2, see also Ref. [18] for further details. Using the above results we can generalize the LO LET by calculating $r$ and $v_{i}$ as functions of the (inverse) scattering length at unphysical values of the pion mass. The resulting predictions are shown by the various thick lines in Fig. 3 for $M_{\pi}=200,300$ and $400 \mathrm{MeV}$. Notice that contrary to chiral EFT extrapolations, we perform calculations with different pion masses as if we lived in different worlds, in which the physical value of the pion mass were $M_{\pi}=200,300, \ldots \mathrm{MeV}$. Thus for each given pion mass the value of $C_{0}$ is adjusted to reproduce the given value of the scattering length used as input.

In order to extend these results to NLO, we need to specify the $M_{\pi}$ dependence of the subleading short-range interaction which is modeled via resonance saturation, see Eq. (3.4). We assume that the $M_{\pi}^{2}$ dependence of the strength $\beta$ of the short-range interaction is within the envelope built by the lines which go through the physical point and describe a $\pm 50 \%$ change in the value of $\beta$ for $M_{\pi}=500 \mathrm{MeV}$. Note that such an estimation is justified for the considered quantities such as $g_{A}$, $F_{\pi}$ and $m_{N}$, as can be seen from Fig. 2.

Our NLO LET predictions for the effective range and the shape parameters viewed as functions of the inverse scattering length are visualized in Fig. 3 by the light-shaded bands. These bands correspond to the variation of $\beta$ at a given value of $M_{\pi}$, as described above. For pion masses below $M_{\pi} \simeq 200 \mathrm{MeV}$, the difference between the predictions based on LO and NLO LET is very small which points at a very rapid convergence of the results. Also, the employed variation of $\beta$ is essentially invisible for such values of the pion mass. For heavier pions, both the differences between the LO and NLO LET as well as the uncertainty in the results associated with the $M_{\pi}$ dependence of $\beta$ start to increase. In particular, for pion masses $M_{\pi} \simeq 400 \mathrm{MeV}$ one can observe large deviations between the shape parameters at LO and NLO which are maximized when one considers the extreme values of the inverse scattering length. On the other hand, in the region of the plateau which corresponds to $\left(a M_{\pi}\right)^{-1} \simeq 0.2-0.7$ the deviations are still not that large. Also the effective range does not change much when one goes from LO to NLO so that the theory might 

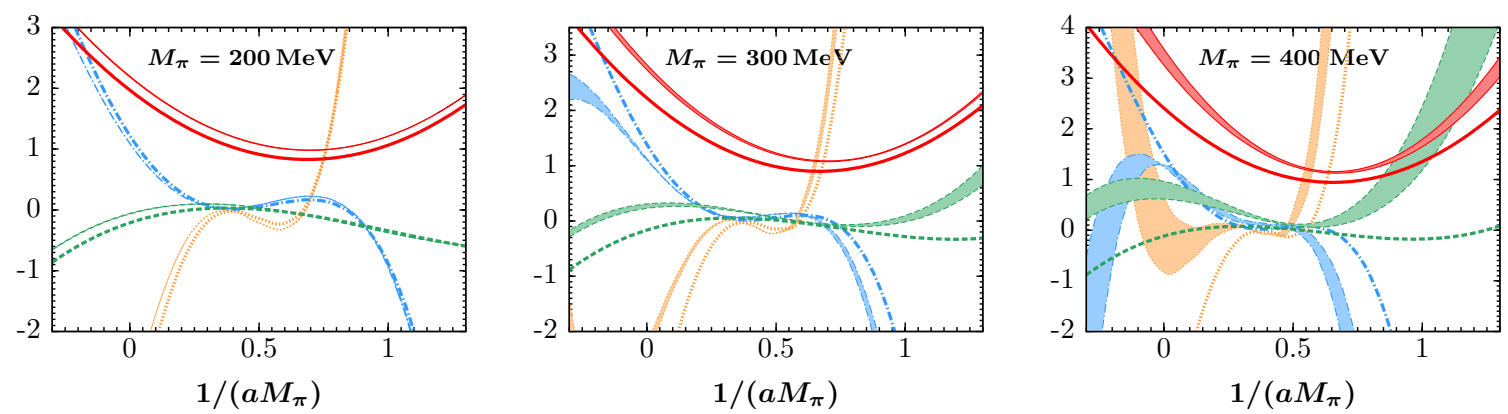

Figure 3: Correlations between the inverse scattering length $a^{-1}$, effective range $r$ and the first three shape parameters $v_{2}, v_{3}$ and $v_{4}$ in the ${ }^{3} S_{1}$ partial wave induced by the one-pion exchange interaction. Various thick lines show the predictions of the LO LET while light-shaded bands (hardly visible for small $M_{\pi}$ ) between thin lines depict the results of NLO LET and reflect the estimated uncertainty due to unknown $M_{\pi}$ dependence of the subleading short-range interaction as explained in the text.

still be convergent and predictive. Eventually, decrease in the predictive power of the LET is to be expected for heavier pions due to the decreasing separation between the soft and hard scales in the problem. On the other hand, for heavy pion masses the $\rho$-meson mass becomes comparable with $2 M_{\pi}$, so that the explicit treatment of two-pion exchanges is not needed.

\section{Implications for lattice QCD}

The LET can provide important consistency checks for the lattice results if several parameters in the ERE are extracted. Unfortunately, not much information on the scattering parameters is available so far $^{4}$. An exception is the work of Ref. [1], which provides, in addition to the binding energies, also the values of the scattering length, effective range and even the first shape parameter at the pion mass of $M_{\pi} \simeq 800 \mathrm{MeV}$. Clearly, such heavy pion masses are beyond the applicability range of the LET. On the other hand, the authors of Ref. [1] conjectured that the quantity $M_{\pi} r$ may behave linearly with the pion mass, as visualized in the left panel of the first row in Fig. 4. Taking this conjecture for granted and using the effective range as an input parameter to adjust the short-range force, we can apply the LET to predict the binding energy and confront it with lattice data. The results of the LET for the binding energy, binding momentum and the first three shape parameters are illustrated in Fig. 4. Interestingly, the general trend of the binding energy extracted from lattice simulations ${ }^{5}$ is nicely described by the LET. Furthermore, due to the left-hand cuts of OPE, the shape parameters appear to be very vivid functions of $M_{\pi}$, the feature which is in principle testable in lattice QCD. In addition, in Ref.[18] we also predicted all parameters in the ERE using the measured binding energies for $M_{\pi}=300 \mathrm{MeV}$ [9] and $M_{\pi}=390 \mathrm{MeV}$ [8]. Further implications will be discussed in the upcoming publications.

\footnotetext{
${ }^{4}$ The analysis of recent NPLQCD results at $M_{\pi} \sim 450 \mathrm{MeV}$ [10] will be discussed elsewhere.

${ }^{5}$ The only exception refers to the data from Ref. [13] which do not support the existence of the bound state for pion masses heavier than the physical one.
} 

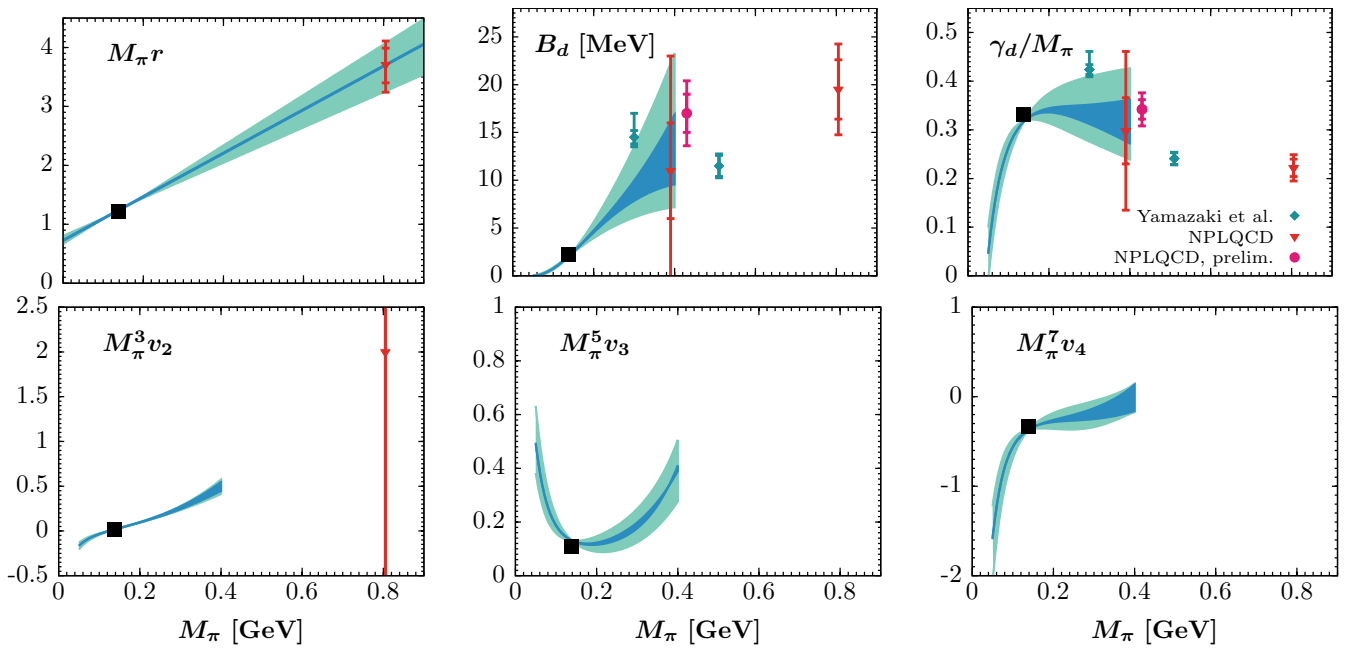

Figure 4: First row left panel: Linear with $M_{\pi}$ interpolation of the quantity $M_{\pi} r$ in the ${ }^{3} \mathrm{~S}_{1}$ partial wave as suggested in Ref. [1]. First row middle and right panels: NLO LET predictions for the pion mass dependence of the deuteron binding energy and the ratio $\gamma_{d} / M_{\pi}$; Second row: NLO LET predictions for the first three shape parameters in the ${ }^{3} \mathrm{~S}_{1}$ partial wave. Dark-shaded bands show our estimation of the uncertainty of the NLO LET due to the unknown $M_{\pi}$ dependence of the subleading short-range interaction, light-shaded bands depict the uncertainty in the linear extrapolation of the effective range used as input.

\section{Summary}

Model independent correlations between the parameters in the effective range expansion caused by long-range interactions are investigated for $\mathrm{NN}$ scattering. They are called low-energy theorems. In particular, using the $\mathrm{NN}$ scattering length in the ${ }^{3} S_{1}$ channel as input, the effective range and the shape parameters predicted from LET at leading order appear to be in good agreement with those extracted from Nijmegen PWA. If data for the effective range are employed in addition to constrain subleading short-range interactions, the shape parameters at NLO are predicted at the few per cent level. The generalization of LET to unphysical pion masses allows one to analyse the correlations induced by one-pion-exchange away from the physical point. Several implications of the analysis for lattice QCD are discussed.

\section{Acknowledgments}

This work is supported by the ERC project 259218 NUCLEAREFT. One of the authors (VB) thanks the DFG (grant GZ: BA 5443/1-1 AOBJ: 616443) for the partial support.

\section{References}

[1] S. R. Beane et al. [NPLQCD Collaboration], Phys. Rev. C 88, no. 2, 024003 (2013)

[2] U.-G. Meißner, Sci. Bull. 60(1), 43 (2015)

[3] E. Epelbaum et al., Phys. Rev. Lett. 110, no. 11, 112502 (2013)

[4] E. Epelbaum, H. Krebs, T. A. Lähde, D. Lee and U.-G. Meißner, Eur. Phys. J. A 49, 82 (2013)

[5] H. Oberhummer, A. Csoto and H. Schlattl, Nucl. Phys. A 689, 269 (2001) 
[6] P. F. Bedaque, T. Luu and L. Platter, Phys. Rev. C 83, 045803 (2011)

[7] J. C. Berengut et al., Phys. Rev. D 87, no. 8, 085018 (2013)

[8] S. R. Beane et al. [NPLQCD Collaboration], Phys. Rev. D 85, 054511 (2012)

[9] T. Yamazaki, K. i. Ishikawa, Y. Kuramashi and A. Ukawa, Phys. Rev. D 92, no. 1, 014501 (2015)

[10] K. Orginos et al., arXiv:1508.07583 [hep-lat].

[11] E. Berkowitz et al., arXiv:1508.00886 [hep-lat].

[12] T. Yamazaki, K. i. Ishikawa, Y. Kuramashi and A. Ukawa, Phys. Rev. D 86, 074514 (2012)

[13] T. Inoue et al. [HAL QCD Collaboration], Nucl. Phys. A 881, 28 (2012)

[14] S. R. Beane, P. F. Bedaque, M. J. Savage and U. van Kolck, Nucl. Phys. A 700, 377 (2002)

[15] S. R. Beane and M. J. Savage, Nucl. Phys. A 717, 91 (2003)

[16] E. Epelbaum, U.-G. Meißner and W. Glöckle, Nucl. Phys. A 714, 535 (2003)

[17] E. Epelbaum and J. Gegelia, PoS CD 12, 090 (2013) [arXiv:1301.6134 [nucl-th]].

[18] V. Baru, E. Epelbaum, A. A. Filin and J. Gegelia, Phys. Rev. C 92, no. 1, 014001 (2015)

[19] T. D. Cohen and J. M. Hansen, Phys. Rev. C 59, 13 (1999); Phys. Rev. C 59, 3047 (1999)

[20] J. V. Steele and R. J. Furnstahl, Nucl. Phys. A 645, 439 (1999)

[21] E. Epelbaum and J. Gegelia, Eur. Phys. J. A 41, 341 (2009)

[22] E. Epelbaum and J. Gegelia, PoS CD 09, 077 (2009).

[23] E. Epelbaum, arXiv:1001.3229 [nucl-th].

[24] J. J. de Swart, C. P. F. Terheggen and V. G. J. Stoks, nucl-th/9509032.

[25] J. M. Blatt and J. D. Jackson, Phys. Rev. 76, 18 (1949).

[26] H. A. Bethe, Phys. Rev. 76, 38 (1949).

[27] B. Midya et al., arXiv:1501.04011 [quant-ph].

[28] H. van Haeringen and L. P. Kok, Phys. Rev. A26, 1218 (1982).

[29] E. Epelbaum and J. Gegelia, Phys. Lett. B 716, 338 (2012)

[30] V. G. Kadyshevsky, Nucl. Phys. B 6, 125 (1968).

[31] V. Baru, E. Epelbaum, A. A. Filin, J. Gegelia and A. V. Nefediev, Phys. Rev. D 92, 114016 (2015)

[32] E. Epelbaum, A. M. Gasparyan, J. Gegelia and H. Krebs, Eur. Phys. J. A 51, no. 6, 71 (2015)

[33] V. G. J. Stoks, R. A. M. Klomp, C. P. F. Terheggen and J. J. de Swart, Phys. Rev. C 49, 2950 (1994)

[34] M. Pavon Valderrama and E. Ruiz Arriola, Phys. Rev. C 72, 044007 (2005).

[35] J. M. Blatt and L. C. Biedenharn, Rev. Mod. Phys. 24, 258 (1952).

[36] D. B. Kaplan, M. J. Savage and M. B. Wise, Nucl. Phys. B 534, 329 (1998)

[37] S. Dürr et al. [Budapest-Marseille-Wuppertal Collaboration], Phys. Rev. D 90, no. 11, 114504 (2014)

[38] C. Alexandrou et al., PoS LATTICE 2013, 292 (2014) [arXiv:1312.2874 [hep-lat]]; PoS LATTICE 2014, 151 (2015) arXiv:1411.3494 [hep-lat]. 